\title{
CHEMICAL SENSORS BASED ON PULSED LASER DEPOSITED MOLECULAR SIEVES
}

\author{
Mary E. Gimon-Kinsel and Kenneth J. Balkus, Jr. \\ Department of Chemistry, The University of Texas at Dallas \\ Richardson, Texas 75083-0688 USA
}

\begin{abstract}
Films of microporous and mesoporous molecular sieves were generated using pulsed laser deposition followed by a brief post hydrothermal treatment. Specifically, films of MeAPO-5 such as CoAPO-5 and Nb-TMS1 were made and subsequently used as the dielectric phase in capacitive-type chemical sensors. The capacitance change of mesoporous Nb-TMS1 based devices to the relative moisture in their environment makes them useful as potential humidity sensors. The CoAPO- 5 based chemical sensors were evaluated for the detection of $\mathrm{N}_{2}, \mathrm{CO}$ and $\mathrm{CO}_{2}$.
\end{abstract}

\section{INTRODUCTION}

Molecular sieves comprise a class of low density metal oxides which possess highly organized one-, two- or threedimensional pores and/or cage-like structures. The size and shape of the pores allow molecules to be discriminated based on steric properties; molecules of similar size can be differentiated based on polarity and acid/base properties. Numerous applications for these selective absorbents as sensors can be envisioned ranging from optical to mass sensitive and capacitive-type devices. These devices would benefit from the fabrication of thin films of molecular sieves.

We have developed a method for molecular sieve thin film generation which utilizes pulsed laser deposition (PLD) and a brief post hydrothermal treatment. This technique has been applied to a variety of porous metal oxide compositions some of which require a sensitizer. We refer to this modification as guest assisted laser ablation (GALA) [1]. We have demonstrated that PLD can be employed to generate thin films of microporous molecular sieves such as UTD-1 [2] and aluminophosphate molecular sieves including $\mathrm{AlPO}_{4}-5$ anc' $\mathrm{MeAPO}(\mathrm{Me}=\mathrm{V}, \mathrm{Mn}$ and $\mathrm{Mg}$ ) [3, 4] . Thin films have been deposited on a variety of substrates including silicon, platinum, titanium nitride (TiN), indium-doped tin oxide (ITO), Mylar and glass. Parallel plate capacitors have been fabricated from these molecular sieve films. The absorption of small molecules (eg., $\mathrm{CO}, \mathrm{N}_{2}, \mathrm{H}_{2} \mathrm{O}, \mathrm{CO}_{2}$ and $\mathrm{NH}_{3}$ ) result in large changes in capacitance which readily allow for the discrimination and detection of such gases at the ppm level.

Recent results for PLD of the microporous molecular sieve CoAPO-5 (pore size $7 \AA$ ) and the mesoporous molecular sieve Nb-TMS1 (pore sizes 22-33 $\AA$ ) will be presented. The CoAPO-5 molecular sieves has an AFI framework topology, and the Nb-TMS1 consists of a hexagonal mesostructure (see Figure 1). This is the first time mesoporous films have been made using this laser deposition technique. The Nb-TMS1 is interesting because it possesses semiconductor properties which might be exploited in electronic devices. Results for the capacitance change of Nb-TMS1 response indicate that they are potential humidity sensors. Preliminary results using CoAPO-5 devices will also be presented. a

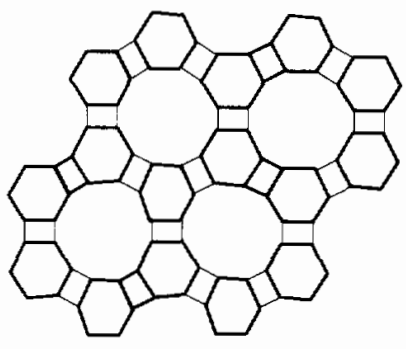

b

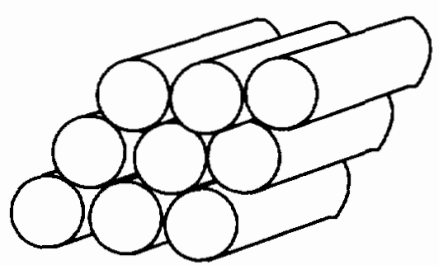

Figure 1. (a) AFI framework structure of COAPO-5 viewed along the 001 direction (b) hexagonal mesostructure of $\mathrm{Nb}-\mathrm{TMS}$.

\section{EXPERIMENTAL DETAILS}

Molecular Sieve Synthesis. The transition metal oxide mesoporous molecular sieve Nb-TMS1 (pore size $22 \AA$ ) was synthesized according to the published procedure. [5] A typical preparation of the synthesis gel involved the mixing of niobium (V) ethoxide (CHEMAT Technology, Inc.) and dodecylamine (Aldrich) with a molar ratio $1: 1.25$. The resulting mixture was heated at $50^{\circ} \mathrm{C}$ for approximately 1 minute, then $10 \mathrm{~mL}$ ethanol followed by $10 \mathrm{~mL}$ deionized water were added which resulted in the precipitation of a white solid. The solid was aged in the supernatant for 72 hours. The white solid and supernatant were loaded into a $23 \mathrm{~mL}$ Teflon lined stainless steel autoclave and heated for 24 hours at $80^{\circ} \mathrm{C}, 24$ hours $100^{\circ} \mathrm{C}$, followed by 7 days at $180^{\circ} \mathrm{C}$. The white product was isolated by suction filtration, washed with deionized water, ethanol and diethylether and dried overnight at room temperature. 
The microporous metal substituted molecular sieve CoAPO-5 was synthesized according to the published procedure. [6] Briefly, the initial synthesis gel was made by combining $1.56 \mathrm{~g}$ of $\mathrm{CoSO}_{4}$ with $1.8 \mathrm{~mL}$ of $85 \%$ phosphoric acid in $10 \mathrm{~mL}$ of deionized water. $2.08 \mathrm{~g}$ of $\mathrm{Al}(\mathrm{OH})_{3}(83.34 \%)$ was added to the cobalt phosphoric acid solution and stirred until homogeneous. 2.9 $\mathrm{mL}$ of triethylammine (TEA) was added to the cobalt phosphoric acid gel and stirred for 1 hour and had a reactant molar ratio of 1.5 TEA:0.8 $\mathrm{Al}_{2} \mathrm{O}_{3}: 1 \quad \mathrm{P}_{2} \mathrm{O}_{5}: 40 \mathrm{H}_{2} \mathrm{O}: 0.4 \mathrm{CoO}$. The gel was transferred to a Teflon lined stainless steel autoclave and heated under static conditions at $200^{\circ} \mathrm{C}$ for 20 hours. The deep blue CoAPO- 5 crystals were centrifuged, washed with deionized water and dried at room temperature.

Pulsed Laser Ablation and Post Hydrothermal Treatment of Films. An illustration of the PLD and post hydrothermal treatment of laser deposited molecular sieve films is shown in Figure 2. A pressed pellet of the molecular sieve was placed in a controlled-atmosphere chamber and irradiated using a Lumonics HyperEx-400 excimer laser ( $248 \mathrm{~nm}\left(\mathrm{KrF}^{*}\right)$, pulse length $14 \mathrm{~ns}$, repetition rate $10 \mathrm{~Hz}$ ). The laser energy was measured using a Scientech pyroelectric head (model 380402) and found to range from 90 to $100 \mathrm{~mJ} /$ pulse. A computer controlled rastoring mirror (Oriel) was used to turn the laser beam $90^{\circ}$ and move the beam across the pellet surface. A focusing lens was employed to decrease the laser beam to a spot size of $\mathrm{ca} .0 .001 \mathrm{~cm}^{2}$. Films were deposited by placing a substrate such as TiN $(500 \AA)$ coated silicon on the heated substrate holder locate approximately $2.5 \mathrm{~cm}$ in front of the pellet. Typical film deposition conditions was as follows: laser power density, $100 \mathrm{MW} / \mathrm{cm}^{2}$; substrate temperature, 150$300^{\circ}$; background oxygen pressure, 250-300 mTorr; deposition time, 10-20 min. Scanning electron microscopy (SEM) was used to examine the morphology and determine the thickness of the deposited films.

The laser deposited Nb-TMS1 and CoAPO-5 films were determined by XRD to be amorphous and partially crystalline, respectively. Both the Nb-TMSI and CoAPO-5 films were then subjected to solution phase hydrothermal treatment. The solution phase treatment of the Nb-TMS1 has been described previously [7]. Briefly, the Nb-TMS1 coated substrate was positioned in the Teflon lined stainless steel autoclave at approximately $60^{\circ}$ angle with the film side facing down. The melted niobium (V) ethoxide and dodecyl amine (molar ratio 1: 1.25) mixture was added to the Teflon liner followed by $20 \mathrm{~mL}$ ethanol. The resulting mixture was allowed to age for 72 hours at room temperature, 4 hours at $90^{\circ} \mathrm{C}, 4$ hours at $140^{\circ} \mathrm{C}$ and 24 hours to 3 days at $180^{\circ} \mathrm{C}$. The film was washed with deionized water, ethanol and diethylether and dried at room temperature. Because solution phase hydrothermal treatment of the CoAPO-5 films resulted in the reorganization of the laser deposited material to yield mixed molecular sieve phases (CoAPO-5 and CoAPO-34) and rapid growth in film thickness, the partially crystalline CoAPO-5 films were used as deposited.

\section{Chemical Sensor Fabrication.}

A capacitive-type sensor was prepared from a hydrothermally treated/template-free $\mathrm{Nb}$ TMS1 film coating a TiN/silicon substrate. The organic template was removed by washing in a solution of 3:1 isopropanol: water acidified with nitric acid to $\mathrm{pH} 1.75$ for 3 hours followed by washing in ethanol. A Au/Pd alloy was patterned onto the film surface using a shadow mask. The device was then heated to $200^{\circ} \mathrm{C}$ to desorb water and immediately transferred to an environmental chamber under a positive pressure of high purity nitrogen where it was allowed to cool to room temperature $\left(24^{\circ} \mathrm{C}\right)$.
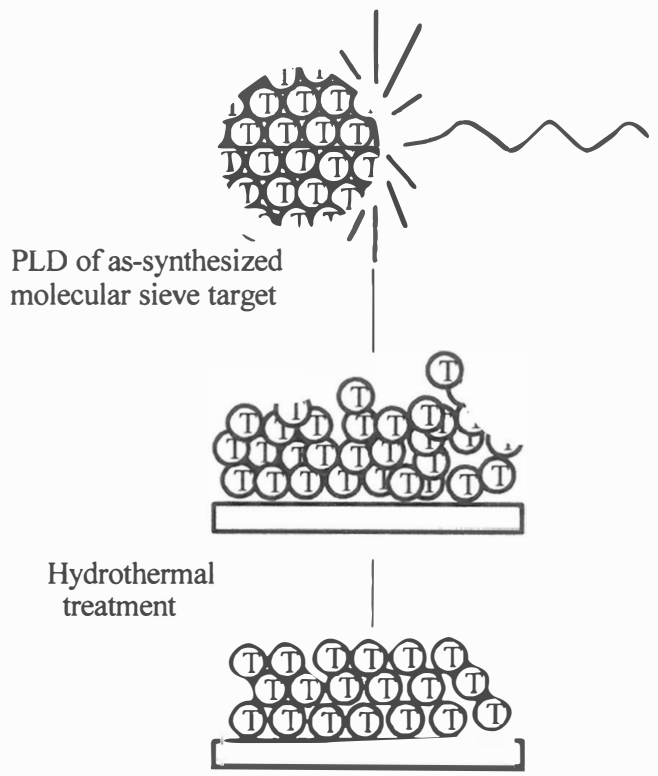

Template removal

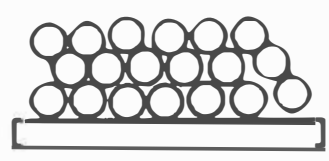

Figure 2. Experimental methodology for pulsed laser deposition and post hydrothermal treatment of molecular sieve films.

The environmental chamber was equipped with two probes by which film plus adsorbate capacitance was measured. One probe made electrical contact with the bare TiN surface and the other probe made contact with a $0.184 \mathrm{~mm}^{2} \mathrm{Au} / \mathrm{Pd}$ electrode. A voltage was applied and the capacitance signal was received by a WayneKerr Precision Component Analyzer 6425. A virtual instrument program written in house LabView for Windows 3.0.1 allowed the applied voltage to be stepped from $0 \mathrm{~V}$ to $0.300 \mathrm{~V}$ and the measured capacitance plotted as a function of applied voltage.

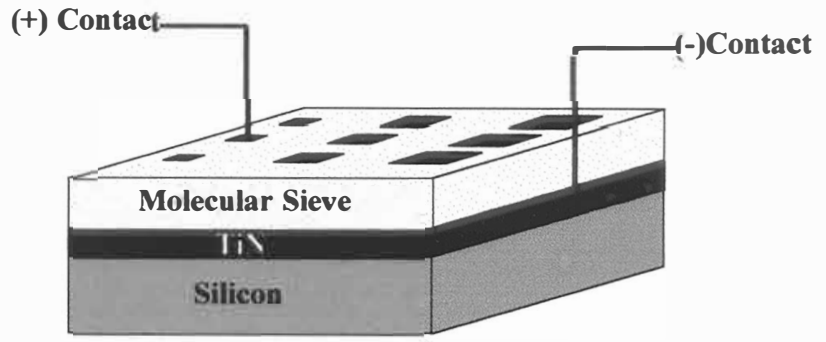

Figure 3. Schematic of molecular sieve thin film based sensor.

\section{RESULTS AND DISCUSSION}

SEM and Transmission electron microscopy (TEM) were used to characterize the laser deposited Nb-TMSI films. In general, SEM images show a continuous, uniform films composed 
of 30-50 nm particulates. The laser deposited film cross-section is shown in Figure 4. This film is on the order of $200 \mathrm{~nm}$ thick which is in the range needed to minimize leakage current while still maintaining significant capacitance changes.

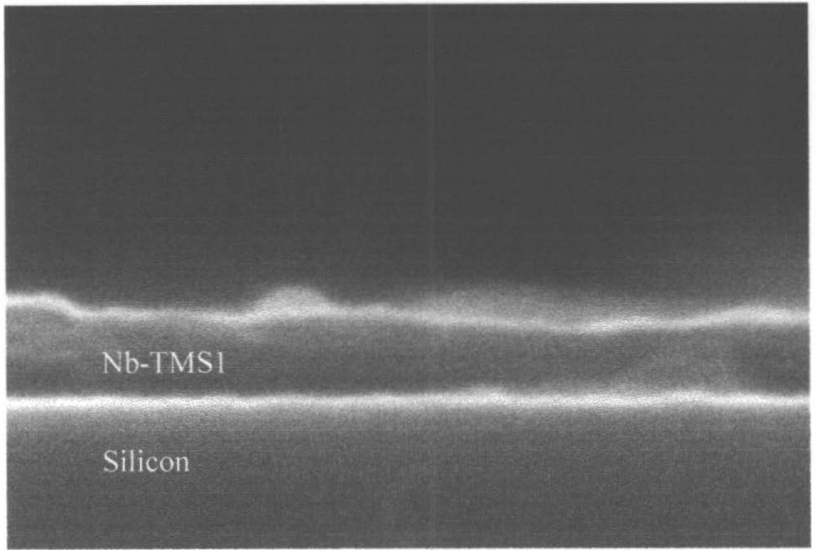

Figure 4. SEM image of the laser deposited Nb-TMS1 films crosssection.

The laser deposited Nb-TMSl appeared to be largely Xray amorphous by powder XRD. However, further examination by TEM revealed areas of crystalline molecular sieve dispersed in an amorphous matrix (see Figure 5).

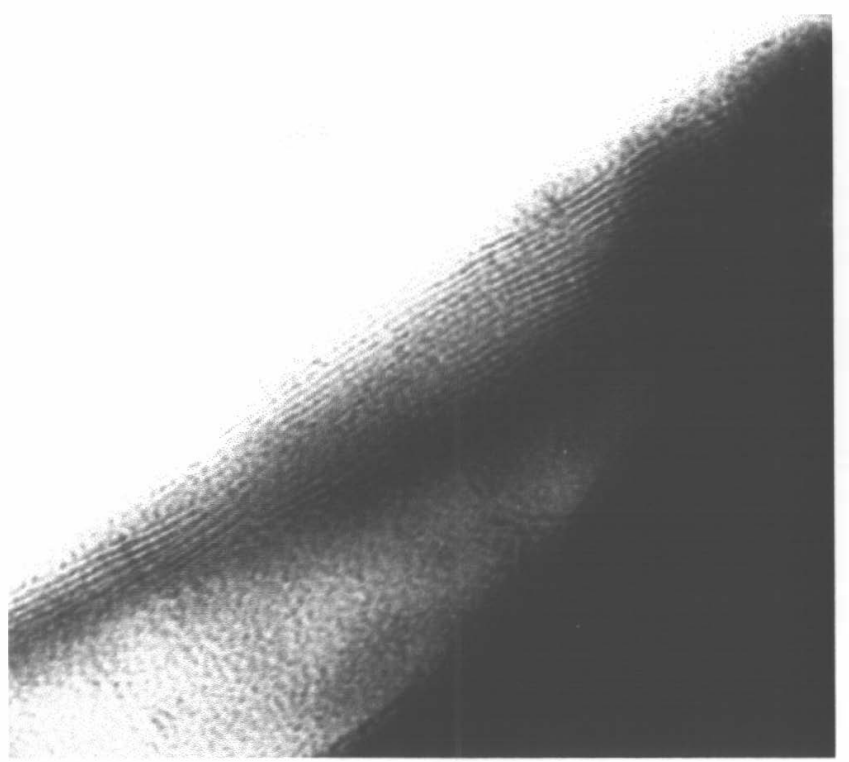

Figure 5. TEM of laser deposited Nb-TMSI film.

We have found that the laser deposited films can be reorganized using a post hydrothermal treatment. Figure 6 illustrates the change in the TEM images that occur following a hydrothermal treatment that was heated at $180^{\circ} \mathrm{C}$ for 24 hours. Larger regions of crystallinity are observed which can be described as having a "worm-like" motif [8]. The XRD pattern also reflects the increase in crystallinity with the appearance of a low angle peak between 23 two-theta. (data not shown).

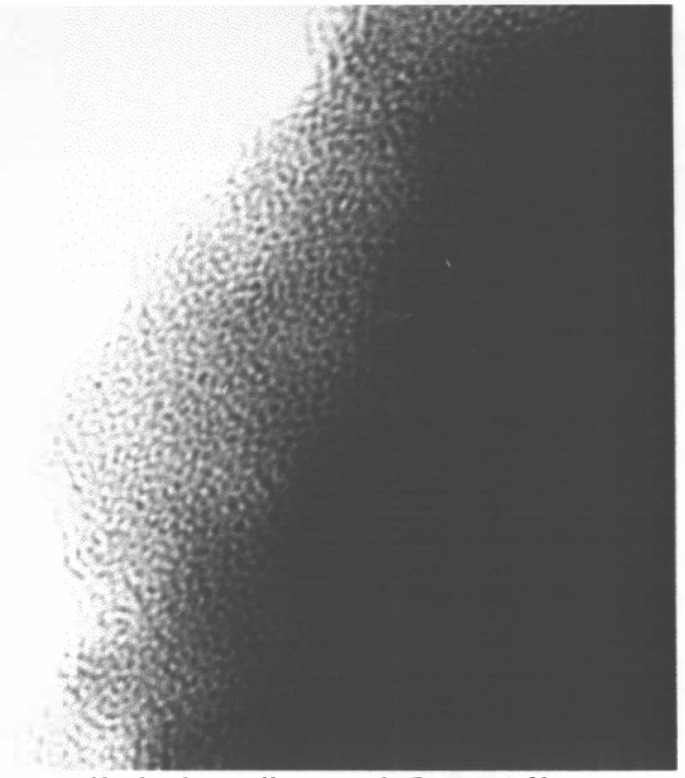

Figure 6. TEM of hydrothermally treated Nb-TMS1 film.

The crystallinity of the laser deposited CoAPO-5 films were shown to range from $\mathrm{X}$-ray amorphous to partially crystalline by powder XRD. The morphology of the laser deposited CoAPO-5 films were very similar to that observed by SEM of the laser deposited Nb-TMSI films.

Capacitive-type chemical sensors were fabricated using the hydrothermally treated/template-free Nb-TMS1 and/or calcined (template-free) CoAPO-5 films as the dielectric phase, the TiN substrate as an electrode and patterned AulPd as the other electrode. By applying a potential between the electrodes, changes in the capacitance were recorded upon adsorption of analyte molecules into the molecular sieve film. We found that the $\mathrm{Nb}$ TMS1 films were particularly sensitive to the relative level of moisture in the environmental chamber. When the Nb-TMS1 based sensor was subjected to a flow of nitrogen containing water vapor, the capacitance measured across the hydrothermally treated film increased as the water was adsorbed. The background capacitance/area measured in flowing high purity nitrogen was on the order of $0.30 \mathrm{nF} / \mathrm{mm}^{2}$. The capacitance/area increased to 5.2 $\mathrm{nF} / \mathrm{mm}^{2}$ when water was introduced into the flowing nitrogen. Because water can moved freely into and out of the pores of the hydrothermally treated film, the capacitance response can be monitored as a function of time. For example, Figure 5 illustrates the reversible response to water of sensors based on mesoporous $\mathrm{Nb}$-TMS1 films which are approximately $300 \mathrm{~nm}$ thick. A repeat cycle (X3) of $90 \%$ humidity followed by a flush of high purity nitrogen was used to determined the reproducibility of the sensor response. During each 10 minute flush with nitrogen, the capacitance returned to its baseline within 1 minute. The average capacitance change varied no more than $+/-2.5 \%$ for the three sequential cycles.

The specificity of the Nb-TMS1 based chemical sensors to water was tested on a variety of gaseous and volatile organics including $\mathrm{CO}_{2}$, methanol and acetone. No measurable response was recorded when the sensor was exposed to $\mathrm{CO}_{2}$. Both methanol and acetone produced a minimal capacitance change $\left(<1 \mathrm{nF} / \mathrm{mm}^{2}\right)$ and therefore would be expected to interfere in the detection of water. The effect of other potential analytes is currently under investigation. 


\section{CONCLUSIONS}

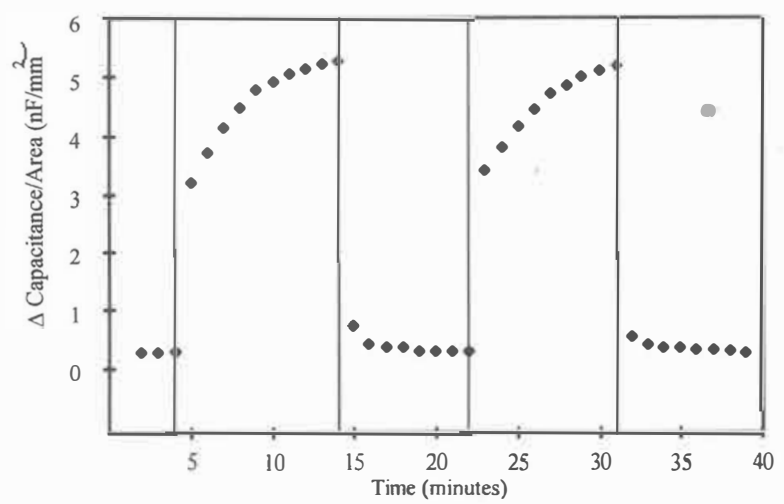

Figure 7. Average reversible response for a capacitive-type $\mathrm{Nb}$ TMS1 chemical sensor to the presence of water vapor in flowing nitrogen (average film thickness $300 \mathrm{~nm}$ ).

A comparison with sensors fabricated from dense $\mathrm{Nb}_{2} \mathrm{O}_{5}$ films indicates that the accessible mesoporosity of the $\mathrm{Nb}$-TMS1 results in capacitance changes which are at least $3 \mathrm{X}$ that observed with the dense phase devices.

The response of the CoAPO- 5 based chemical sensors was measured in the presence of a number of analytes including $\mathrm{N}_{2}, \mathrm{CO}_{2}$ and $\mathrm{CO}$. The CoAPO-5 based devices were found to be selective and sensitive to $\mathrm{CO}$ and $\mathrm{CO}_{2}$ (see Figure 8). The background capacitance measured in the presence of high purity nitrogen was on the order of $10^{-14} \mathrm{~F} / \mathrm{mm}^{2}$. When $\mathrm{CO}_{2}$ is added to the nitrogen flow, the capacitance increases to $10^{-9} \mathrm{~F} / \mathrm{mm}^{2}$. A change in capacitance of $>3$ orders of magnitude is observed when the sensor is exposed to $\mathrm{CO}$ indicating CoAPO- 5 is a viable sensor for $\mathrm{CO}$ even in the presence of $\mathrm{CO}_{2}$. The difference in the sensor response is dramatic for molecules of similar size and shape. The kinetic diameter of $\mathrm{CO}(3.8 \AA)$ is larger than $\mathrm{CO}_{2}(3.3 \AA)$.

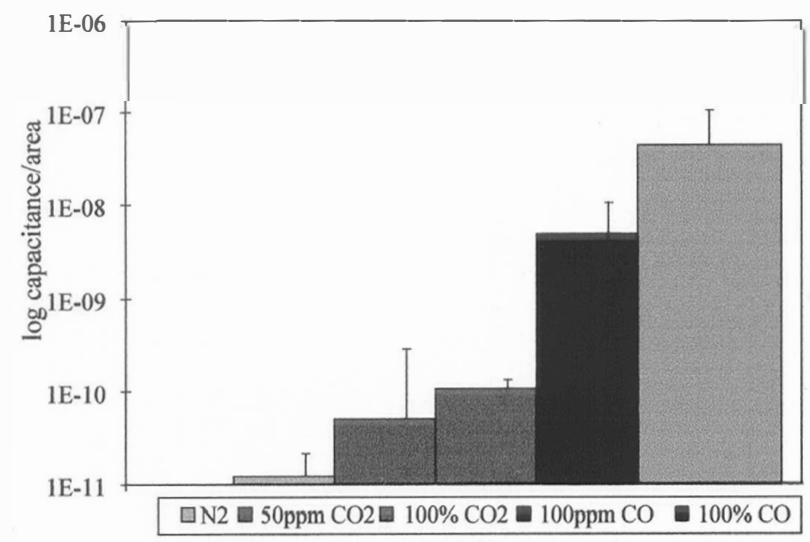

Figure 8. Capacitance response of CoAPO-5 based sensor to $\mathrm{CO}_{2}$ and $\mathrm{CO}$ (film $227 \mathrm{~nm}$ thick).
Continuous films of mesoporous and microporous molecular sieves have been prepared using pulsed laser deposition. PLD followed by a solution phase or vapor phase post hydrothermal treatment can be used to generate crystalline $\mathrm{Nb}$ TMS1 and CoAPO-5 films, respectively. Capacitance-type chemical sensors can then be fabricated using these films as the dielectric phase. It was shown that chemical sensors based on $\mathrm{Nb}$ TMS1 films are sensitive to the relative level of moisture in the environment which makes such sensors useful as potential humidity sensors. In contrast, CoAPO-5 is not sensitive to water but exhibits large capacitance changes upon exposure to $\mathrm{CO}$. The thermal stability of the molecular sieves $\left(>400^{\circ} \mathrm{C}\right)$ suggest that sensors based on these materials may be particularly useful for the detection of combustion gases. We anticipate that the compositional variances available for both mesoporous and microporous molecular sieves will lead to more selective sensors as well as other applications for these thin films.

\section{ACKNOWLEGMENTS}

We wish to thank the Texas Advancd Technology Program and Kenneth Hoffman for assistance with the TEM.

\section{REFERENCES}

1. M. E. Gimon-Kinsel, T. Muñoz, Jr., A. Ayala, and K. J. Balkus, Jr. (manuscript in preparation).

2. K. J. Balkus, Jr., T. Muñoz, Jr., and M. E. Gimon-Kinsel "Pulsed Laser Deposition of Zeolite UTD-1: Evidence for Oriented Film Growth", Chem. Mater., 10, 464 (1998).

3. K. J. Balkus, Jr., L. J. Ball, M. E. Gimon-Kinsel, J. M. Anthony, and B. E. Gnade "A capactivite -type chemical sensor that employs VAPO-5, MnAPO-5 and MAPO-36 molecular sieves as the dielectric phase", Sensors \& Actuators B, 42, 67 (1997).

4. K. J. Balkus, Jr., L. J. Sottile, B. E. Gnade, and J. M. Anthony “ A capacitance type chemical sensor based on $\mathrm{AlPO}_{4}-5$ molecular sieves", Chem. Mater., 9, 380 (1997) and unpublished results.

5. D. M. Antonelli, A. Nakahira, and J. Ying "Ligand-assisted liquid crystal templating in mesoporous niobium oxide molecular sieves" Inorg. Chem., 35, 3126 (1996).

6. K. J. Chao, S. P. Sheu, S. H. Chen, J. C. Lin, and J. Lievens "Molecular Sieves: Synthesis of Microporous Materials," M. L. Occelli and H. Ratson (Eds) (1992), p.317.

7. M. E. Gimon-Kinsel and K. J. Balkus, Jr. " Mesoporous molecular sieve thin films", to be presented at the First International Symposium on Mesoporous Molecular Sieves., Baltimore, MD (1998).

8. S. A. Bagshaw and T. J. Pinnavaia, "Mesoporous Molecular Sieves," Angew. Chem. Int. Ed. Engl., 35, 1102 (1996). 